Alexander Adam and Carlo J. De Luca

J Neurophysiol 90:2919-2927, 2003. doi:10.1152/jn.00179.2003

You might find this additional information useful...

This article cites 29 articles, 11 of which you can access free at:

http://jn.physiology.org/cgi/content/full/90/5/2919\#BIBL

This article has been cited by 8 other HighWire hosted articles, the first 5 are:

Time required for the restoration of normal heavy exercise VO2 kinetics following prior heavy exercise

M. Burnley, J. H. Doust and A. M. Jones

J Appl Physiol, November 1, 2006; 101 (5): 1320-1327.

[Abstract] [Full Text] [PDF]

Decomposition of Surface EMG Signals

C. J. De Luca, A. Adam, R. Wotiz, L. D. Gilmore and S. H. Nawab

J Neurophysiol, September 1, 2006; 96 (3): 1646-1657.

[Abstract] [Full Text] [PDF]

Rotation of Motoneurons During Prolonged Isometric Contractions in Humans

P. Bawa, M. Y. Pang, K. A. Olesen and B. Calancie

J Neurophysiol, September 1, 2006; 96 (3): 1135-1140.

[Abstract] [Full Text] [PDF]

Evaluation of plateau-potential-mediated 'warm up' in human motor units

A. J. Fuglevand, A. P. Dutoit, R. K. Johns and D. A. Keen

J. Physiol., March 15, 2006; 571 (3): 683-693.

[Abstract] [Full Text] [PDF]

Establishment of a protocol to test fatigue of the trunk muscles

G Corin, P H Strutton and A H McGregor

Br. J. Sports Med., October 1, 2005; 39 (10): 731-735.

[Abstract] [Full Text] [PDF]

Medline items on this article's topics can be found at http://highwire.stanford.edu/lists/artbytopic.dtl on the following topics:

Physiology .. Muscle Fibers

Medicine .. Fatigue

Physiology .. Humans

Updated information and services including high-resolution figures, can be found at:

http://jn.physiology.org/cgi/content/full/90/5/2919

Additional material and information about Journal of Neurophysiology can be found at: http://www.the-aps.org/publications/jn

This information is current as of June 7, 2007.

Journal of Neurophysiology publishes original articles on the function of the nervous system. It is published 12 times a year (monthly) by the American Physiological Society, 9650 Rockville Pike, Bethesda MD 20814-3991. Copyright (C) 2005 by the American Physiological Society. ISSN: 0022-3077, ESSN: 1522-1598. Visit our website at http://www.the-aps.org/. 


\title{
Recruitment Order of Motor Units in Human Vastus Lateralis Muscle Is Maintained During Fatiguing Contractions
}

\author{
Alexander Adam and Carlo J. De Luca \\ NeuroMuscular Research Center and Department of Biomedical Engineering, Boston University, Boston, Massachusetts 02215
}

Submitted 26 February 2003; accepted in final form 30 June 2003

\begin{abstract}
Adam, Alexander and Carlo J. De Luca. Recruitment order of motor units in human vastus lateralis muscle is maintained during fatiguing contractions. J Neurophysiol 90: 2919-2927, 2003; 10.1152/jn.00179.2003. Motor-unit firing patterns were studied in the vastus lateralis muscle of five healthy young men [21.4 $\pm 0.9(\mathrm{SD}) \mathrm{yr}]$ during a series of isometric knee extensions performed to exhaustion. Each contraction was held at a constant torque level, set to $20 \%$ of the maximal voluntary contraction at the beginning of the experiment. Electromyographic signals, recorded via a quadrifilar fine wire electrode, were processed with the precision decomposition technique to identify the firing times of individual motor units. In repeat experiments, whole-muscle mechanical properties were measured during the fatigue protocol using electrical stimulation. The main findings were a monotonic decrease in the recruitment threshold of all motor units and the progressive recruitment of new units, all without a change of the recruitment order. Motor units from the same subject showed a similar time course of threshold decline, but this decline varied among subjects (mean threshold decrease ranged from 23 to $73 \%$ ). The mean threshold decline was linearly correlated $\left(R^{2} \geq 0.96\right)$ with a decline in the elicited peak tetanic torque. In summary, the maintenance of recruitment order during fatigue strongly supports the notion that the observed common recruitment adaptations were a direct consequence of an increased excitatory drive to the motor unit pool. It is suggested that the increased central drive was necessary to compensate for the loss in force output from motor units whose muscle fibers were actively contracting. We therefore conclude that the control scheme of motor-unit recruitment remains invariant during fatigue at least in relatively large muscles performing submaximal isometric contractions.
\end{abstract}

\section{N T R O D U C T I O N}

The force level of a voluntary muscle contraction is controlled through two mechanisms: recruitment of motor units and modulation of the firing rate of active motor units. Both recruitment and firing rate modulation occur according to a set of rules in response to a common excitatory drive, which greatly reduces the computational burden on the CNS (De Luca and Erim 1994; De Luca et al. 1982b). The recruitment order follows the size principle first reported by Henneman et al. (1965a,b), where size refers to some measure of motor unit size, such as motoneuron soma diameter, axon conduction velocity, or muscle-fiber twitch response. At a given submaximal voluntary force level, the mean firing rates of motor units are inversely proportional to their recruitment thresholds, such that earlier recruited, lowthreshold motor units maintain higher firing rates than later

Address for reprint requests and other correspondence: C. J. De Luca, NeuroMuscular Research Center, 19 Deerfield St., Boston, MA 02215 (E-mail: cjd@bu.edu). recruited, higher threshold units. This phenomenon was observed during constant as well as force-varying contractions in a variety of limb muscles (De Luca and Forrest 1973; De Luca et al. 1982a,b, 1996; Kanosue et al. 1979; Person and Kudina 1972; Tanji and Kato 1973) and later termed the "onion skin" property (De Luca and Erim 1994). The term stems from the fact that mean firing rates plotted as a function of time-when contraction force is increased, held constant, and then decreased-resemble the layers of the skin of an onion. Other independent investigators have confirmed this phenomenon in human and animal studies (Hoffer et al. 1987; Masakado et al. 1995; Rose and McGill 2001; Stashuk and de Bruin 1988).

There are conflicting reports as to whether the recruitment properties of motor units are invariant with fatigue. For example, several researchers have described recruitment and silencing of motor units during prolonged contractions (Fallentin et al. 1993; Kato et al. 1981; Person 1974; Westgaard and De Luca 1999; among others). Yet our understanding of the stability of recruitment thresholds during sustained contractions remains ambiguous. Only a few studies have monitored changes to recruitment thresholds during repeated submaximal contractions and these have produced conflicting results. Christova and Kossev (1998) reported a decrease in the mean recruitment threshold with fatigue, while Carpentier et al. (2001) and Enoka et al. (1989) reported that the recruitment threshold during their fatigue test was not affected homogeneously but instead showed a wide range of adaptations among their motor-unit sample populations. Taken together, these reports might inspire the notion of divergent behavior among concurrently active motor units in fatigued muscle and implicitly postulate a departure from the ordered recruitment scheme of the nonfatigued state. Such altered recruitment would imply that the size principle and the onion skin property were violated during fatigue, implying that the control of motor units was in some way altered during fatigue. Consequently, we performed this study with the purpose of systematically investigating changes to motor-unit recruitment behavior in a muscle during long-duration muscle activity. By simultaneously tracking the firing patterns of a group of motor units in each trial, the current study improves upon previous reports that were limited to observations on single motor units. Our approach provides a direct assessment of recruitment order.

\footnotetext{
The costs of publication of this article were defrayed in part by the payment of page charges. The article must therefore be hereby marked "advertisement" in accordance with 18 U.S.C. Section 1734 solely to indicate this fact.
} 
METHODS

\section{Subjects}

Five healthy men, age 20-22 years [21.4 \pm 0.9 (SD) yr], reporting no known neuromuscular disorders, participated in this study. All subjects read and signed an informed consent form approved by the Institutional Review Board at Boston University.

\section{Torque and surface electromyographic recordings}

Subjects were seated in a special chair to restrain hip movement and fix the dominant leg at a knee angle of $60^{\circ}$ flexion. Isometric knee extension torque was measured via a lever arm and a pad positioned against the tibia $3 \mathrm{~cm}$ above the medial malleolus. Subjects controlled the knee extension torque via a feedback display on a computer screen. A parallel bar bipolar surface electrode (DE2.1, Delsys) placed on the vastus lateralis (VL) muscle of the dominant leg provided an indication of global muscle activity. In addition, synergist and antagonist muscle activity was monitored via additional surface electrodes placed on vastus medialis, rectus femoris, and biceps femoris. Torque and surface electromyographic (EMG) signals were band-pass filtered from DC- $-100 \mathrm{~Hz}$ and $10 \mathrm{~Hz}$ to $1 \mathrm{kHz}$, respectively, and digitized at $2 \mathrm{kHz}$.

\section{Motor-unit recordings}

A quadrifilar fine wire electrode was used to record intramuscular EMG signals from the VL muscle of the dominant leg. The placement of the electrode was in close proximity to the surface electrode, which was positioned in the distal third of the VL muscle. Each electrode consisted of four 50- $\mu \mathrm{m}-$ diam nylon-coated $\mathrm{Ni}-\mathrm{Cr}$ wires glued together and cut to expose only the cross section of the wires (De Luca and Adam 1999). The fine wire electrode was inserted into the muscle via a 25-gauge disposable hypodermic needle, which was withdrawn leaving the electrode in place. This arrangement permits the experimenter to reliably record motor units with minimal discomfort to the subject and provides stable recordings of up to $1 \mathrm{~h}$ (Westgaard and De Luca 1999). Three combinations of wire pairs were selected and differentially amplified to yield three separate EMG channels. The signals were band-pass filtered $(1-10 \mathrm{kHz})$ to accentuate the differences in the wave shapes, sampled at $50 \mathrm{kHz}$, and stored on a PC for offline data analysis.

\section{Protocol}

First, subjects performed three brief ( $3 \mathrm{~s}$ ) maximal knee extension contractions, the highest of which was used to establish the initial maximal voluntary contraction (MVC) torque. Subjects were then asked to trace displayed trajectories by exerting the appropriate amount of extension torque. Several practice trajectories were given to ensure that each subject was able to smoothly grade the torque output. Overall, subjects performed 7-10 contractions separated by at least $3 \mathrm{~min}$ of rest time before proceeding to the fatigue series. This practice maneuver was necessary to produce the consistent force profiles for each set of the fatiguing contractions. The fatigue protocol consisted of repeated contractions of a constant target level, which was set to $20 \%$ of the initial MVC value. (The fact that the absolute force was maintained constant for each contraction will become relevant in the interpretation of the results.) Each cycle started with a 5-s ramp up to 50\% MVC and a brief hold phase after which the torque was decreased to the target value of $20 \%$ MVC and held constant for $50 \mathrm{~s}$ (Fig. 1). The ramp at the beginning of each cycle served two purposes: it allowed for the repeated measurement of recruitment thresholds under comparable conditions in progressive contractions, and it revealed the recruitment thresholds of motor units that were initially not active during the constant part of the contraction. At the end of each cycle, the muscle activity was decreased at the same rate (10\% MVC/s) as the initial ramp and a brief $(6 \mathrm{~s})$ period of rest allowed the subject prepare for the next contraction. The sequence of contractions was performed until the subject could no longer maintain the target level. Strong verbal encouragement was given to the subjects when the torque traces repeatedly dipped below the target value of $20 \%$ MVC by more than $1 \%$ MVC (5\% of the target value). The trial was terminated by the experimenter at the end of a cycle, when the dips in the torque occurred at a rate of more than two per $10 \mathrm{~s}$ of constant target torque.

\section{Electrical stimulation}

Only three of the original five subjects were available to be tested with transcutaneous electrical stimulation. For these three subjects, the voluntary fatigue protocol was repeated on

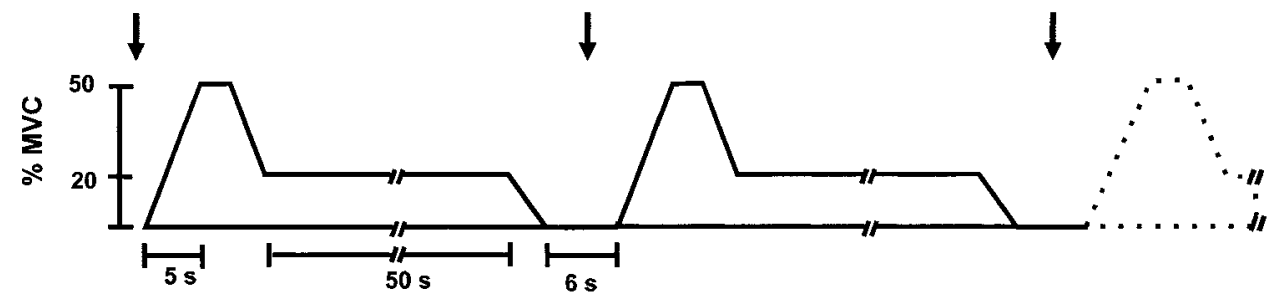

FIG. 1. Two cycles of the knee extension torque trajectory traced by the subjects during the fatigue protocol. Each cycle consisted of a brief ( $5 \mathrm{~s}$ ) ramp up to a medium torque level, followed by a long (50 s) plateau at a lower, constant torque level. Rest time between successive contractions was $6 \mathrm{~s}$. All torque values were scaled to the maximum voluntary contraction (MVC) torque, which was determined at the beginning of each experimental session. The torque level for the fatigue protocol was set to $20 \%$ MVC for the long plateau, that of the initial ramp and hold to 50\% MVC. All subjects practiced tracing the trajectory during the first half of the experimental session to achieve smooth torque transitions at $10 \% \mathrm{MVC} / \mathrm{s}$. Three subjects repeated the fatigue protocol on a separate occasion. During this second test, no intramuscular EMG signals were acquired, but electrical stimulation was applied to measure the contractile properties of the VL muscle. $\downarrow$, timing of electrical stimulation, which was administered before, after, and in between the voluntary contractions. 
a separate occasion without intramuscular EMG recordings. The VL muscle was stimulated using two self-adhesive surface electrodes $(4.5 \times 4.5 \mathrm{~cm})$ placed proximally and distally over the lateral aspect of the thigh. Optimal electrode placement was determined by fixing the current intensity and repeatedly eliciting a single muscle twitch as the proximal electrode position was varied. The electrode was secured to the skin at the location, which produced the largest twitch amplitude. During the testing electrical square-wave pulses $(0.2 \mathrm{~ms}$ in duration) from a constant current stimulator were administered at supramaximal (110\%) intensity. Maximum intensity was achieved by gradually increasing the stimulation current until no further increase in twitch amplitude could be observed. Supramaximal stimulation ensured that the largest possible muscle mass was activated for a given electrode location. Constant area of the elicited M wave obtained from the VL surface electrode was taken as evidence that the same or a similar population of muscle fibers were stimulated during the fatigue protocol. The $\mathrm{M}$ wave was recorded by blanking the output stage of the EMG amplifiers at the time of the stimulation pulse. Because the muscle was activated through the proximal stimulation electrode, positioned on the proximal motor point, the elicited $\mathrm{M}$ wave, recorded $18-20 \mathrm{~cm}$ distally, occurred at a sufficient latency to the stimulation pulse. The knee extension torque during maximal voluntary effort and in response to a test stimulation train was examined $3 \mathrm{~min}$ before and immediately after the voluntary fatigue protocol. The test stimulation consisted of a brief train of 11 stimuli at $50 \mathrm{~Hz}$. Throughout the voluntary fatigue protocol, the test train was administered during each 6-s rest period between successive voluntary contraction (Fig. $1 \downarrow$ ). In two subjects, the $50-\mathrm{Hz}$ train was followed (1-s delay) by a single stimulus to measure the twitch response. This revealed the time course of the muscle response to single and tetanic stimulation as fatigue progressed. In previous studies of quadriceps muscle fatigue, the $50-\mathrm{Hz}$ stimulation rate has been shown to produce a fused muscle contraction (Bigland-Ritchie et al. 1986; Dolmage and Cafarelli 1991; Vøllestad et al. 1997).

\section{Analysis}

The precision decomposition technique (De Luca and Adam 1999; LeFever and De Luca 1982) was used to separate the intramuscular EMG signals into the constituent action potential trains, thus reconstructing individual motor unit firing trains. Briefly, this technique uses rule-based algorithms to identify action potentials and allocate them to motor units via template matching, template updating, calculation of firing probabilities, and resolution of superpositions. In some cases, including the ones reported in this paper, the decomposition can be done with 100\% accuracy (LeFever et al. 1982; Mambrito and De Luca 1984).

The similarity of the motor units' action potential shapes, on each of the three channels, across successive contractions provided confirmation that the same units were tracked across contractions. This was a critical validation and only contractions in which the identified motor units provided this confirmation are reported. In addition, for motor units that had more than 50 firings during a contraction, a surface EMG signature was computed by spike-triggered averaging the surface EMG signal detected from the VL muscle. Based on the premise that the surface EMG signature of a motor unit cannot abruptly change shape during isometric contractions, the consistency of the surface EMG signature from one contraction to the next was taken as a validation of the motor unit tracking derived from the intramuscular electrode.

The recruitment threshold of a motor unit was calculated as the torque level at the first consistent firing (next firing within $250 \mathrm{~ms}$ ) of the motor unit. This way of calculating the threshold avoided erroneous threshold values due to glitches in the torque signal and singular motor-unit firings. The recruitment thresholds were analyzed in detail during the first, middle, and last contraction of the fatigue protocol for all subjects. Intermediate contractions were processed with the automatic precision decomposition routines to facilitate tracking of individual motor units.

\section{R E S U L T S}

Intramuscular EMG signals on five subjects were successfully analyzed for motor-unit recruitment during intermittent, long-duration knee extension contractions. The number of motor units identified for each subject ranged from 9 to $12(11.0 \pm$ 1.7) for a total of 55 distinct motor units. The number of successive contractions ranged from 6 to $10(8.20 \pm 2.05)$ and did not show a significant dependency on pre-fatigue knee extension torque MVC values $\left(R^{2}=0.42, P>0.05\right)$, which ranged from 139.59 to $220.89 \mathrm{Nm}(198.42 \pm 33.57 \mathrm{Nm})$. The amplitude of surface EMG signals recorded from the VL and its agonist muscles increased from the first to the last contraction. Minimal surface EMG activity was observed in the biceps femoris during the fatigue protocol.

An example of knee torque and motor unit firing train data from one subject is given in Fig. 2. The figure shows the first, middle, and last contraction in a series of 10 repeated contractions. In each of the three graphs, the solid line represents the isometric knee extension torque, normalized to the MVC value measured at the beginning of the experimental session. Motorunit firing times, represented as vertical bars, are linked to the torque record by a common time axis. To the right of each graph, insets depict snapshots of the motor-unit action potential shapes that were used to identify individual firing trains. Each shape consists of the motor-unit action potential waveform in three channels. The shapes were sufficiently different from one another and only changed gradually over time, thus allowing the tracking of continuously active and the identification of newly recruited motor units during the fatigue protocol. In this figure, we see the recruitment of new motor units in contraction 5 during the initial torque peak [motor units (MUs) 9-11] as well as recruitment during the constant torque plateau (MUs 3-6). Two additional motor units were recruited by the end of the contraction series amounting to 12 motor units tracked for this subject. The recruitment of new motor units during the 50\% MVC ramp and the progressive recruitment during the $20 \%$ MVC plateau were seen in all subjects. Thus three observations could be made from these and similar plots of motor-unit firing times. First, a greater number of motor units were recruited to generate the ramp to $50 \% \mathrm{MVC}$ as the contraction number increased and fatigue accumulated. Second, an increasing number of motor units were continuously active in subsequent contractions (i.e., did not turn off when the torque level was 

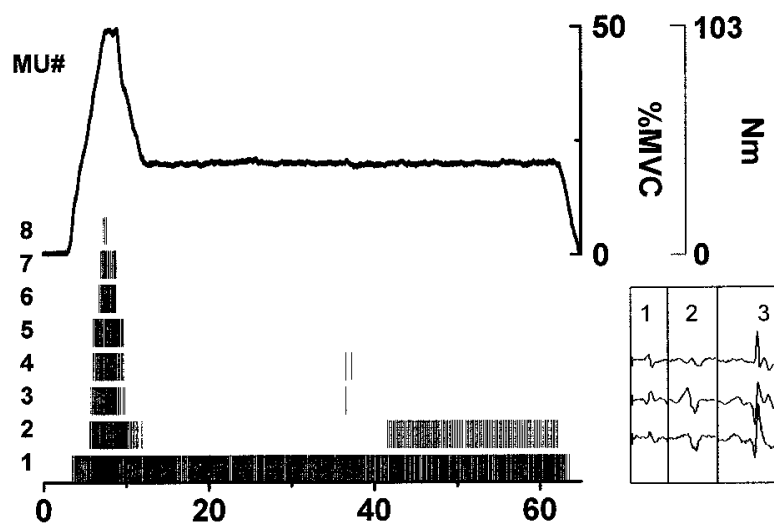

Contraction \# 1

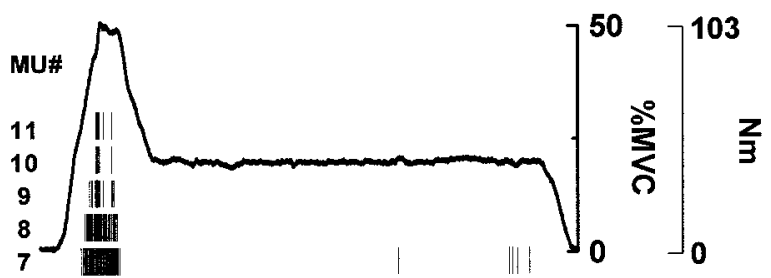

FIG. 2. The first (top), middle (middle),

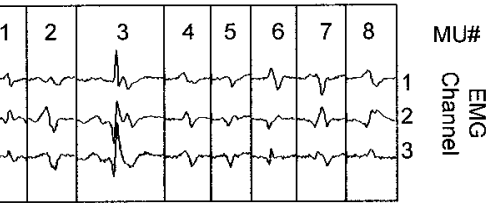

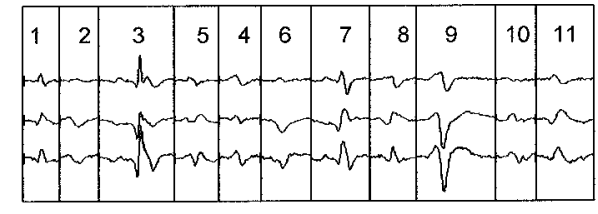

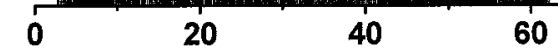

Contraction \# 5

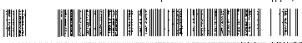

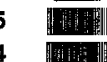

IN

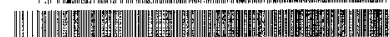

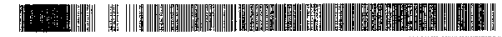

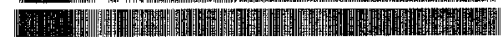

20

40

60
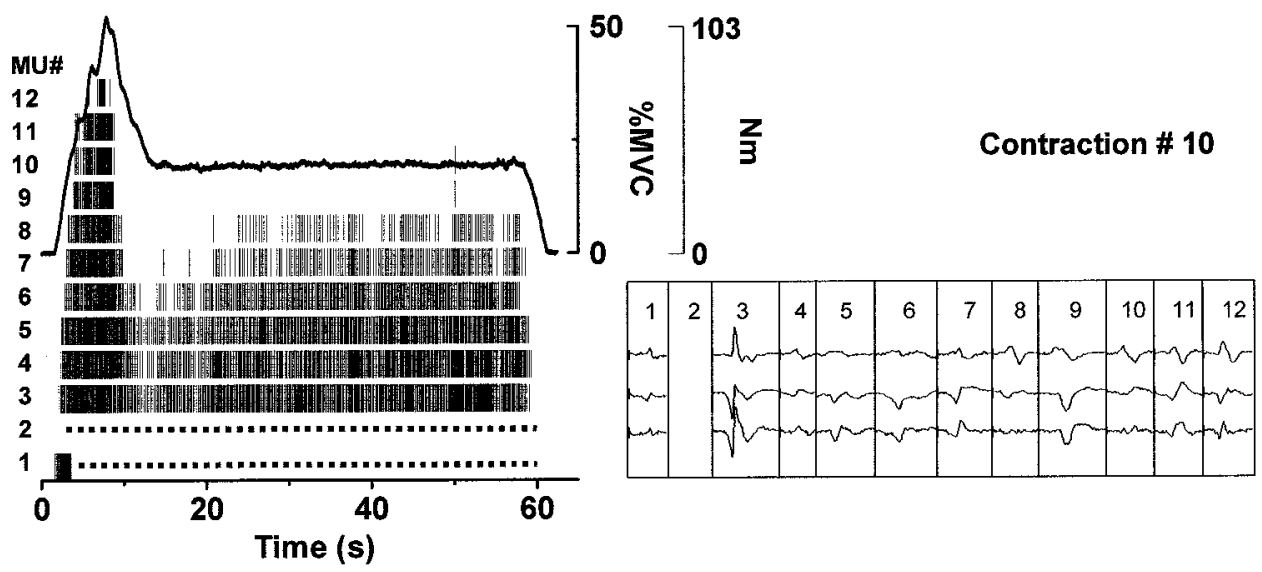

and last (bottom) cycle in a series of 10 successive VL contractions in 1 subject. Shown are isometric knee extension torque (solid line, right vertical axis), both in absolute (Nm) and relative measures (\% MVC), and motor unit firing times, represented as vertical bars. Insets: motor-unit action potential shapes used to identify individual firing trains. Numbers above the 3-channel EMG signatures correspond to the assigned motor-unit numbers in the bar plots. Dotted lines indicate that a motor-unit shape could no longer be reliably detected. See text for further details. decreased from 50 to $20 \%$ MVC). Third, a greater number of motor units were active to hold the torque constant during the $20 \%$ plateau. The number of motor units active during the initial torque peak and the lower torque plateau are tabulated in Table 1.

Figure 3 provides a detailed view of the ordered recruitment of motor units during the initial ramp in the torque traces. The torque curves and the motor-unit firing times are shown side by side on an expanded time scale for the same three contractions as depicted in Fig. 2. Note the common axis for all three torque traces and the separate time axes for the motor-unit firings. This arrangement facilitates comparison of recruitment thresholds among contractions. The figure shows that to generate the same rate of increase and the same absolute torque level, motor units were recruited earlier (i.e., at lower torque levels), as well as in larger numbers (newly recruited) during successive contractions. The decrease in the recruitment threshold, which was observed in all motor units, is highlighted for one of the motor units (MU 3).

The recruitment thresholds as a function of normalized endurance time for the first subject are given in Fig. 4A. Apart from the first, middle, and last contractions that were shown in Fig. 2, additional contractions were analyzed for this subject at intermediate time intervals to highlight the consistency in the motor-unit behavior. Note that all motor units exhibited a similar monotonic decrease in their recruitment threshold as the contraction series progressed. All the while, the order of recruitment remained strikingly unchanged from that of the initial contraction. During subsequent contractions, newly recruited motor units always became active during the initial ramp phase at higher torque levels than all previously active motor units.

For the remaining four subjects, motor-unit recruitment patterns were analyzed at the beginning, middle, and end of the 
TABLE 1. Number of active motor units observed during the peak and the plateau phase of the first, middle, and last contraction in the fatigue series

\begin{tabular}{lcccccccc}
\hline \hline & \multicolumn{2}{c}{ First } & & & \multicolumn{2}{c}{ Middle } & & \multicolumn{2}{c}{ Last } \\
\cline { 2 - 3 } \cline { 8 - 9 } Subject & Plateau & Peak & & Plateau & Peak & & Plateau & Peak \\
\hline$A$ & 2 & 8 & & 5 & 11 & & 7 & 12 \\
$B$ & 2 & 5 & & 3 & 7 & & 8 \\
$C$ & 5 & 11 & & 8 & 12 & & 8 & 12 \\
$D$ & 3 & 8 & & 4 & 11 & & 4 & 11 \\
$E$ & 2 & 4 & & 3 & 11 & & 3 & 12 \\
Average & 2.8 & 7.2 & & 4.6 & 10.4 & & 5.6 & 11 \\
Percent & 25.5 & 65.5 & & 41.8 & 94.5 & & 50.9 & 100.0 \\
\hline
\end{tabular}

A motor unit was said to be active during the plateau phase of the contraction if it fired continuously for a minimum of $10 \mathrm{~s}$. The motor-unit count includes units whose recruitment threshold could not be reliably determined for all contractions.

fatigue series (Fig. 4, B-E). At least three motor units were tracked from start to end in each subject with additional units recruited during the fatigue task. The same pattern of a progressive decline in the threshold as well as the recruitment of additional, initially higher threshold units was observed in all subjects. Motor units that could not be followed to the last contraction did not drop out, but exhibited a dramatic decrease in amplitude and/or shape changes consistent with that caused by the movement of the electrode away from the action potential source. When this reduction in amplitude and modification in shape became severe, the computer algorithms could no longer identify the action potentials as belonging to a particular motor unit. Overall, 26 motor units could be followed from the start of the fatigue protocol to the end. An additional 16 units that had been recruited midway through the contraction series were successfully tracked from there on. A significant positive correlation between the initial and the final value of the recruitment thresholds $\left(R^{2}=0.51, P<0.001, n=26\right)$ reinforced the above-mentioned observation that the recruitment order was preserved. A similar result was obtained when recruitment thresholds were not expressed as a percentage of each subject's MVC value, but in absolute torque values.

To compare recruitment changes for different motor units, the recruitment threshold was normalized by the torque value at which the motor unit was initially recruited. Only motor units that were active from the beginning of the fatigue test were used for this analysis. Figure 4, $a-e$, shows plots of the normalized recruitment thresholds as a function of endurance time for each subject. Motor units recorded from the same subject always showed a similar time course of threshold decline. The decrease in motor unit threshold was monotonic and, overall, not determined by the initial value of the recruitment threshold. Thus for pooled data, the initial threshold was a poor predictor of the relative change [(initial - final)/initial] in threshold $\left(R^{2}=0.14, P>0.05, n=26\right)$. This overall lack of a significant correlation was not consistently observed in individual data sets. For the first three subjects (Fig. 4, $a-c$ ), the relative threshold change was proportional to the initial threshold value. Correlation coefficients were high $(0.88,0.90$, 0.84 ) but only statistically significant in subjects $A$ and $C$, who had the largest number of observations $(n=7)$.

While the change in the recruitment threshold torques was consistent within individual subjects, the degree of decline varied across subjects. Figure 5 presents an overlay of the average recruitment threshold data from each of the subjects. The normalized recruitment thresholds for each subject in Fig. 4 were averaged at each measurement time to produce this figure. The decrease in the mean recruitment threshold at the endurance limit ranged from 23 to $73 \%$ and was not proportional to endurance time $\left(R^{2}=0.08, P>0.05, n=5\right)$ or maximum leg extension torque $\left(R^{2}=0.62, P>0.05, n=5\right)$.

To test if changes in motor unit threshold occurred in concert with changes in the mechanical properties of the muscle, the fatigue protocol was repeated in three of the subjects in the volunteer group. Electrical stimulation was administered before, in between, and after the voluntary contractions. No motor-unit recordings were made. The number of repeated contractions was 10,22, and 7 with corresponding MVC values

\section{Contraction \#1}

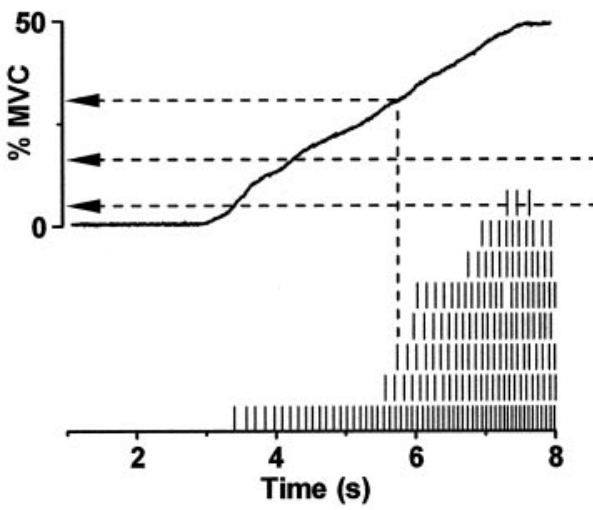

Contraction \#5

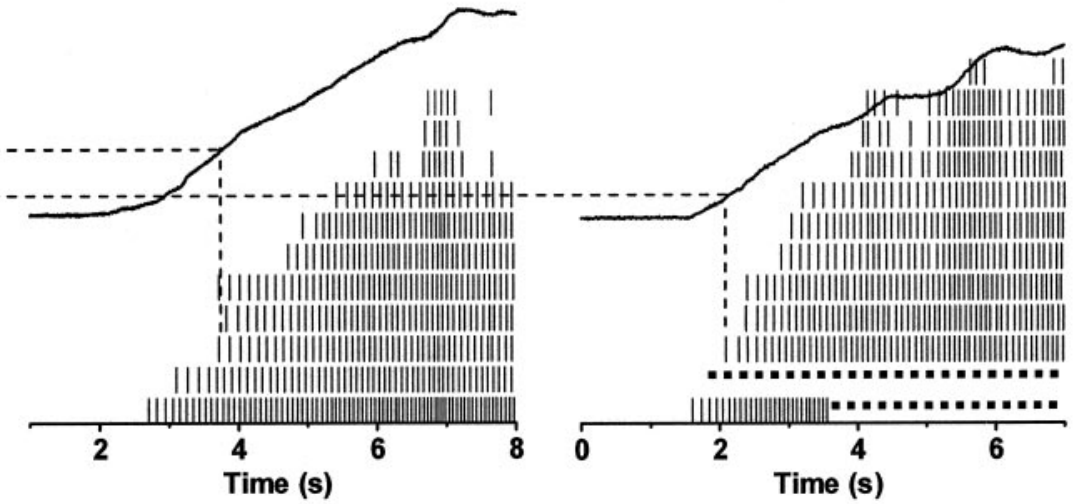

Contraction \#10

Detailed view of the recruitment of motor units during the initial ramp phase of the first, middle, and last contraction in a series of 10 consecutive contractions performed to exhaustion (same contractions as in Fig. 2). Shown are 7-s segments of knee extension torque (solid lines) and corresponding motor unit firing times (vertical bars). Torque curves are scaled to the MVC torque and plotted on the same axis. Note the separate time axis for each of the 3 contractions. The recruitment threshold of a motor unit was measured as the value of the knee torque at the time of the first firing. Dotted lines with arrowheads indicate the decrease in the recruitment threshold from the first to the last contraction for 1 motor unit (MU 3). 


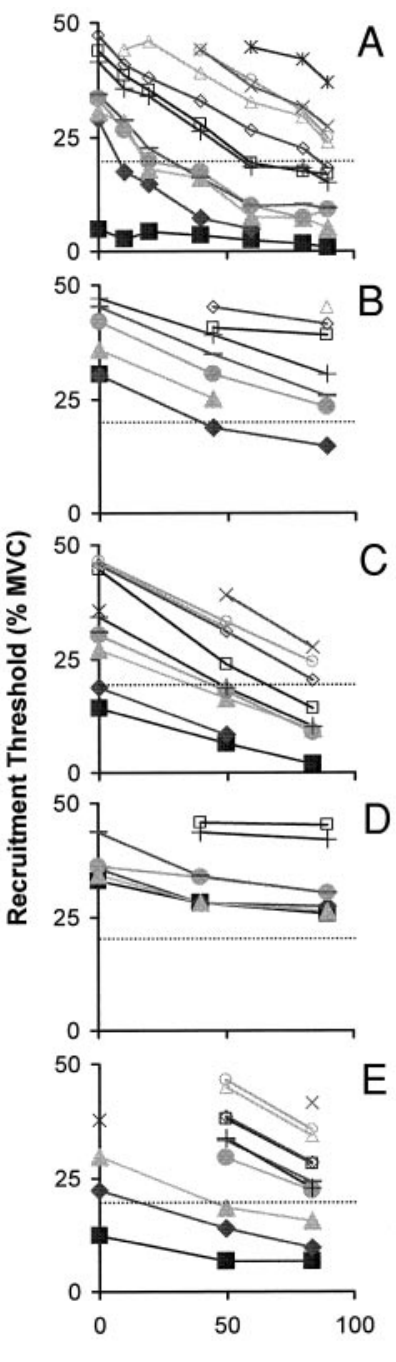

$\%$ Endurance Time

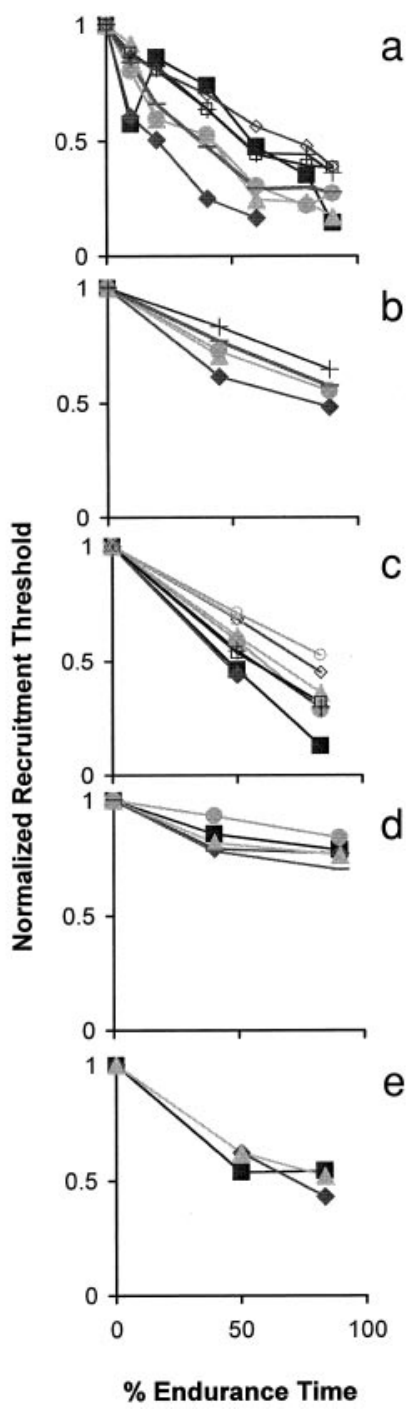

$\%$ Endurance Time

FIG. 4. A: the recruitment threshold, expressed as a percentage of the MVC torque, for 12, concurrently active motor units in a vastus lateralis (VL) muscle plotted as a function of normalized endurance time. Different symbols and shadings denote different motor units. Dotted horizontal lines mark the target torque level that was sustained for $50 \mathrm{~s}$ in each of the contractions. Data shown are from the same subject as in Fig. 2. In addition to contractions 1, 5, and 10 , intermediate cycles were analyzed in this subject to demonstrate the monotonic decline in motor-unit thresholds. Because recruitment thresholds were assessed at the beginning of each 1-min contraction cycle, normalized endurance time values for this subject ranged from 0 (1st contraction) to $90 \%$ (10th contraction). Note the consistent decrease of recruitment torque with increasing endurance time in all motor units. $B-E$ : recruitment threshold data for each of the remaining 4 subjects. Only the first, middle, and last contractions were analyzed. Symbols as in $A . a-e$ : the recruitment threshold, normalized to initial value, of motor units active from the beginning of the fatigue task. Each of the plots shows the recruitment data as a function of normalized endurance time for motor units recorded simultaneously in 1 subject. Note the similarity in the threshold decline in all motor units from any given subject.

of $300.20,191.91$, and $249.13 \mathrm{Nm}$. After the last contraction, the MVC values had declined to $249.22,173.73$, and 185.84 $\mathrm{Nm}$, or 83,90 , and $74 \%$ of the respective initial values. In contrast to the moderate decrease in maximal voluntary torque, the electrically stimulated contractions declined much more dramatically. Figure 6 shows the series of torque responses that stimulation trains at $50 \mathrm{~Hz}$ elicited in one of the subjects (subject $C$, cf. Fig. $4 C$ ). Note the continuous decrease in the

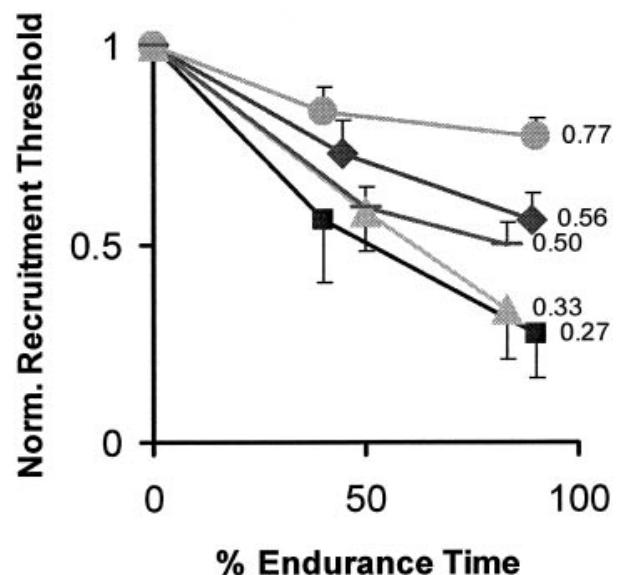

FIG. 5. The mean normalized recruitment threshold as a function of normalized endurance time for motor units from each of 5 subjects. Data points are shown at endurance times corresponding to the first, middle, and last contraction for each subject. Only motor units active from the beginning of the fatigue task were analyzed. Each symbol corresponds to 1 subject in Fig. 4: square, $A$; diamond, $B$; triangle, $C$; circle, $D$; bar, $E$. Error bars denote $1 \mathrm{SD}$. Numbers at the end of each curve denote the mean threshold value for the last contraction.

peak torque from the first measurement immediately before start of the fatigue protocol ( $0 \%$ endurance time) to the last measurement immediately after the last voluntary contraction (100\% endurance time). In this case, the tetanic torque declined to $33 \%$ of the initial value. A qualitatively similar result was obtained for the other subjects, where peak torque at $50 \mathrm{~Hz}$ declined to 53 and $51 \%$ of the initial value. In contrast to the amplitude changes, the duration of the tetanic torque response, as measured by the time to reach peak torque and the half relaxation time $(1 / 2 \mathrm{RT})$, changed less consistently in all subjects. The final time to peak torque measured 108, 92, and $110 \%$ of the initial value, whereas the $1 / 2 \mathrm{RT}$ measured 114 , 95 , and $104 \%$. Thus the area under the tetanic torque response curve displayed a similar decline with fatigue as the peak torque value. In addition to the $50-\mathrm{Hz}$ stimulation, single twitch measurements were available from two subjects. The decrease in twitch amplitude and area was greater than that for the

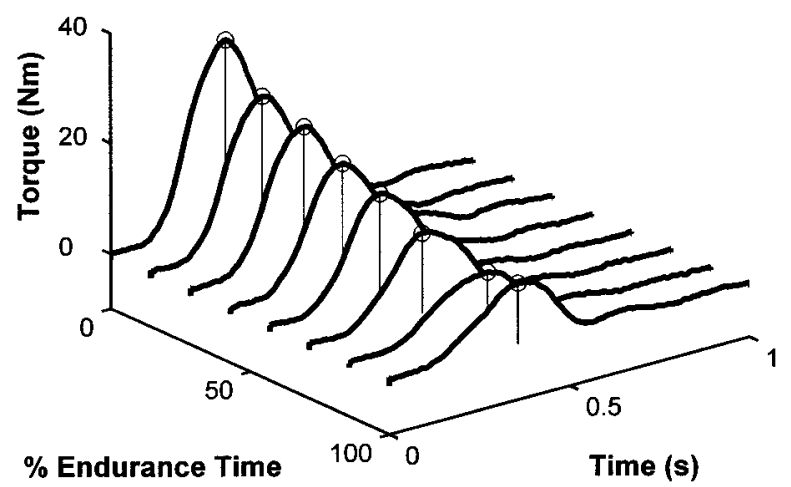

FIG. 6. Series of electrically evoked contractions in the VL muscle recorded during the fatigue task. Electrical stimulation consisted of 11 supramaximal current pulses at $50 \mathrm{~Hz}$. The waterfall plot shows the amplitude $(z$ axis) and contraction time ( $y$ axis) for individual torque responses as a function of normalized endurance time on the ( $x$ axis). The torque response at $0 \%$ endurance time was elicited immediately before the 1 st voluntary contraction, the one at $100 \%$ endurance time immediately after the last contraction. Note the steady decline in peak torque (indicated by superimposed circles and drop lines) as the percentage of endurance time increases. 
$50-\mathrm{Hz}$ tetanus. The twitch amplitude fell to 48 and $15 \%$ of the initial value for subjects $A$ and $C$, respectively. The twitch area decreased to 39 and $13 \%$ of the initial value. As for the tetanic response, the contraction time of the twitch was affected little, measuring 84 and $78 \%$ of the initial value at the end of the endurance time. The $1 / 2 \mathrm{RT}$ decreased to $88 \%$ in subject $A$. Measurements of 1/2RT in subject $C$ became somewhat erratic toward the end of the fatigue series due to large decrease in twitch amplitude. Little change in 1/2RT was observed until the $50 \%$ mark.

\section{I S C U S S I O N}

The main results from this study of submaximal fatiguing contractions in the VL muscle were the monotonic decline in the recruitment threshold of all motor units and the progressive recruitment of new motor units without change to the recruitment order. These results clearly demonstrate that in the VL muscle the ordered recruitment of motor units, commonly observed in nonfatigued skeletal muscle, was also maintained during fatigue. Unlike a recent report by Carpentier et al. (2001), no difference in recruitment threshold decline was seen between relatively high ( $>25 \%$ MVC) and relatively low $(<25 \%$ MVC) threshold motor units. Furthermore and in contrast to earlier reports (Enoka et al. 1989; Carpentier et al. 2001), the change in recruitment threshold was not proportional to the initial threshold value nor did the threshold of any motor unit increase with fatigue. As we can clearly and without ambiguity see in Fig. 4A, the recruitment order of the motor units was not altered in any perceivable manner during the sequential contractions, including the very last one that led to exhaustion.

Both Carpentier and Enoka obtained their measurements from the first dorsal interosseous muscle. The difference in the findings might possibly be attributable to different properties of the two muscles, but in our estimation, this is unlikely. Both muscles are mixed muscles without a striking preponderance of either type I or type II fibers. Data for the fiber type distribution in the first dorsal interosseous muscle are sparse. An autopsy study by Johnson et al. (1973) reported the mean proportion of type I fibers from six subjects to be $57.4 \%$ in the first dorsal interosseous and 46.9 and $37.8 \%$ in the VL for the deep and superficial part of the muscle, respectively. It is conceivable that the difference in the observed firing patterns might also be attributable to the different force protocols employed in the previous studies. Both Carpentier et al. (2001) and Enoka et al. (1989) used intermittent contractions at 50\% MVC, while the current investigation was sustained target torque plateaus at $20 \%$ MVC. However, the duty cycle (time on target/total time of cycle) in the previous studies ranged from 0.5 to 0.7 , whereas ours was 0.9. The combination of higher target levels and lower duty cycles in the previous studies and the lower target level and greater duty cycle in our study would complement each other and produce comparable rates of fatigue. Support for this argument can be found in the similarity of mean endurance times of $8.35 \mathrm{~min}$, reported by Carpentier and colleagues, and $9.29 \mathrm{~min}(8.2$ contractions $\times 68 \mathrm{~s} /$ contraction $)$ in the current study.

If differences in muscle type and force level may not account for the discrepancies in motor-unit firing behavior between the current and previous studies, then what might? We suspect that some of the heterogeneity of motor unit adaptations reported by the two previous studies on the first dorsal interosseous arose from pooling motor-unit firing patterns from different trials and different subjects. Because of the time-varying firing behavior of motor units and the high inter-subject variability, averaging of motor-unit data from separate trials inevitably obscures the ordered firing pattern of any given trial. This limitation especially applies to any study that attempts to make inferences about the control of the motor-unit pool but relies primarily on recordings of one or two motor units at a time. As the current study clearly demonstrated, the degree of motorunit threshold decline varied threefold between subjects (Fig. 5) but was very consistent within each subject.

The particular torque profile employed in this study allowed us to ask the question as to what extent the recruitment threshold decline was influenced by the activation history of a motor unit. To do so, we separated the motor units into two groups: motor units that were active from the first contraction on $(n=$ 26 ), and motor units that were newly recruited at $50 \%$ endurance time $(n=15)$. None of the newly recruited motor units participated in the $20 \%$ MVC plateau phase of the contractions. These units only fired briefly during the initial torque peak and were silent for $90 \%$ of the duration of the contractions. In contrast to this intermittent activity pattern, the majority of the motor units in the first group were either active during the torque plateau from the start (continuous activity) or became active during the plateau as the fatigue series progressed. Thus motor units in the two groups experienced a very different activation history. Yet both groups showed a decrease in recruitment threshold from the $50 \%$ to the $100 \%$ endurance time mark. On the average, the relative change in threshold [(mid$\mathrm{dle}-$ final $) /$ middle] was $-0.24 \pm 0.14$ for the newly recruited motor units and $-0.33 \pm 0.21$ for those active from the beginning. Because of the limited number of observations for the newly recruited motor unit group, no statistical comparison between groups was performed. In any of the subjects, no striking difference between continuously active and newly recruited motor units was apparent (cf. Figs. 2 and 5). We therefore conclude that differences in activation history among the motor units of a given VL muscle were not reflected in the common decrease in recruitment thresholds. This finding disfavors changes to motoneuron intrinsic properties as a likely candidate for the observed recruitment adaptations. The uniformity of the threshold decline found in this study points to a source that is extrinsic to the individual differences in the properties of motor units but common to all the motor units, such as the common drive (De Luca and Erim 1994).

Because the threshold of all motor units in the VL muscle did in fact decrease, the current study fails to explain the findings of Westgaard and De Luca (1999). In that study, the authors presented examples of motor-unit substitution in the trapezius muscle, each coinciding with brief periods of inactivity during otherwise constant low-level muscle activation. It was then speculated that, as a result of continuous activation, motor units might experience an increase in their recruitment threshold to the point where they become less susceptible to excitation than initially higher threshold motor units. In the $\mathrm{VL}$, recruitment thresholds decreased irrespective of whether a motor unit was active from the beginning or progressively recruited during the contraction sequence. Differences in the experimental paradigm (4 vs. $20 \%$ MVC contraction level, surface 
EMG vs. torque feedback) and differences in the motor-unit control properties of the investigated muscles might account for the different results. Evidence that low-threshold motor units in the trapezius muscle can exhibit firing behavior, which differs from that of commonly investigated limb muscles, was recently provided by the same authors (Westgaard and De Luca 2001). Although these differences between muscles are worthy of consideration, we do not believe that they would cause the timedependent recruitment behavior of the trapezius to be different than that of the VL reported herein. We now view the motor-unit substitution observed by Westgaard and De Luca (1999) as more likely to be caused by changes in the proprioceptive feedback from the muscle spindles when the force output of the muscle decreases sharply as was the case in their experiments.

The amplitude of the twitch and tetanic torque response in our subjects was consistently reduced at the endurance limit (cf. Fig. 6) but the contractile speed (CT, 1/2RT) was only minimally affected. These results are in line with previous reports that also employed a brief $50-\mathrm{Hz}$ tetanic train to measure changes in the contractile response associated with fatiguing submaximal contractions, in particular those that resulted in endurance times similar to the current study (Garland et al. 1997; Vøllestad et al. 1997). From a functional perspective, the observed decline in peak torque indicates a reduction in the force capacity of the muscle, since the decreased twitch amplitude was not offset by an increase in twitch duration.

The reduced force capacity of the muscle, as assessed by brief tetanic stimulation at $50 \mathrm{~Hz}$, provides a simple explanation for the observed recruitment changes. Subject B, who showed a reduction in peak tetanic torque by $46 \%$ at the end of the fatigue series, had a decrease in the mean recruitment threshold by $44 \%$. Similarly, the peak tetanic torque and the mean recruitment threshold in subject $C$ both decreased by $67 \%$. The similarity in the decline of peak tetanic torque and of mean motor-unit recruitment threshold was further examined by correlating these parameters at corresponding endurance times. Figure 7 presents a plot of mean normalized recruitment threshold as a function of normalized peak tetanic torque. Three data points are shown for each of the subjects, corresponding to measurements from the beginning, middle, and end of the fatiguing contraction series. In all subjects, the reduction in the peak torque is linearly correlated with the decrease in mean recruitment threshold $\left(R^{2}=1.00,1.00,0.96\right)$. In two of the subjects, the fit regression lines essentially have a slope of 1 and an intercept of 0 . Although these data do not prove a causal relationship between changes in muscle force output and changes to motor unit recruitment, we do believe that the results are not accidental. There are two other observations of motor-unit recruitment within the contractions that are consistent with the inter-contraction recruitment behavior. Figure 2 provides clear evidence that even within contractions motor units are recruited in the early part of the constant torque phase. In subsequent contractions, the newly recruited motor units receive greater excitation, and some of the motor units that became inactive in the early contractions remain active in subsequent contractions, as cataloged in Table 1.

All the observations of the motor-unit behavior may be explained by the mechanism earlier proposed by De Luca et al. (1996). As the force capacity of continuously active muscle fibers declines progressively, increased excitation is required to keep the muscle output constant. The increased excitation

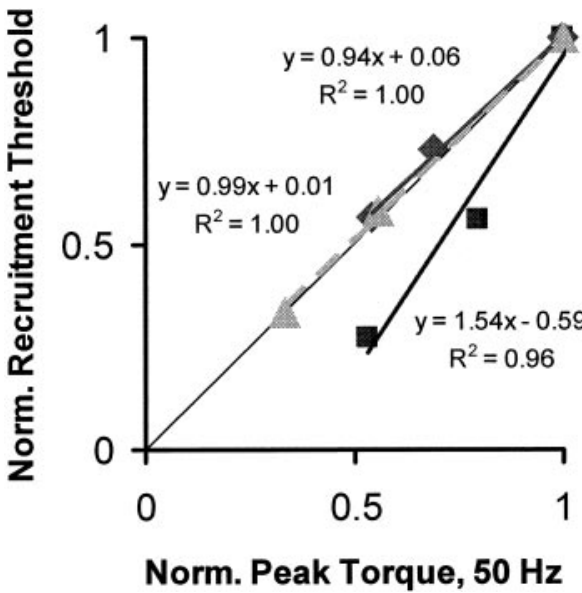

FIG. 7. Correlation of mechanical changes in VL muscle with motor-unit recruitment threshold decline during the fatigue protocol. Mean normalized recruitment threshold of concurrently active motor units is plotted against normalized peak torque of the tetanic force response. For each subject, three data points, corresponding to measurements at or near 0,50 , and $100 \%$ endurance time, are shown. In 2 subjects, data points lie very close to the line of identity (thin solid line), reflecting a very high correlation $\left(R^{2}=1.00\right)$ between the reduction in peak torque and the decline in mean recruitment threshold. In the 3rd subject, the rate of decrease in mean recruitment threshold was greater than the rate of decrease in peak torque, but the relationship was essentially linear $\left(R^{2}=0.96\right)$ as in the other 2 subjects. Symbols and shading as in Fig. 5.

produces the recruitment of additional motor units. The recruited motor units thus become active at a lower torque level than their initial threshold, and the recruitment threshold continues to decrease in subsequent contractions as the force production of the active motor units continues to decrease.

In summary, this study in the VL muscle provided evidence that the recruitment threshold of all observed motor units declined during submaximal fatiguing contractions without any perceivable change to the recruitment order. This result implies that the control scheme used by the CNS to recruit motor units for force production remains invariant during fatigue at least in isometric contractions at low torque levels.

The assistance of M. Sullivan and J. Wu in processing the data is greatly appreciated. We are grateful to Drs. Z. Erim, S. H. Nawab, R. M. Roark, and H. F. Voigt for helpful comments and discussions.

\section{IS C L O S URES}

This work was supported by a Bioengineering Research Partnership Grant 1R24HD-38585 from the National Center for Medical Rehabilitation Research of the National Institute of Child Health and Human Development.

\section{REFERENCES}

Bigland-Ritchie B, Cafarelli E, and Vøllestad NK. Fatigue of submaximal static contractions. Acta Physiol Scand 128: 137-148, 1986.

Carpentier A, Duchateau J, and Hainaut K. Motor unit behaviour and contractile changes during fatigue in the human first dorsal interosseous. J Physiol 534: 903-912, 2001.

Christova $\mathbf{P}$ and Kossev A. Motor unit activity during long-lasting intermittent muscle contractions in humans. J Appl Physiol 77: 379-387, 1998.

De Luca CJ and Adam A. Decomposition and analysis of intramuscular electromyographic signals. In: Modern Techniques in Neuroscience Research, edited by Windhorst U and Johansson H. Heidelberg, Germany: Springer, 1999, p. 757-776.

De Luca CJ and Erim Z. Common drive of motor units in regulation of muscle force. Trends Neurosci 17: 299-305, 1994. 
De Luca CJ, Foley PJ, and Erim Z. Motor unit control properties in constant-force isometric contractions. J Neurophysiol 76: 1503-1516, 1996

De Luca CJ and Forrest WJ. Some properties of motor unit action potential trains recorded during constant force isometric contractions in man. Kybernetik 12: 160-168, 1973.

De Luca CJ, LeFever RS, McCue MP, and Xenakis AP. Behavior of human motor units in different muscles during linearly varying contractions. J Physiol 329: 113-128, 1982a.

De Luca CJ, LeFever RS, McCue MP, and Xenakis AP. Control scheme governing concurrently active human motor units during voluntary contractions. J Physiol 329: 129-142, 1982b.

Dolmage $\mathbf{T}$ and Cafarelli $\mathbf{E}$. Rate of fatigue during repeated submaximal contractions of human quadriceps muscle. Can J Physiol Pharmacol 69: 1410-1415, 1991.

Enoka RM, Robinson GA, and Kossev AR. Task and fatigue effects on low-threshold motor units in human hand muscle. J Neurophysiol 62: 1344-1359, 1989.

Fallentin N, Jørgensen K, and Simonsen EB. Motor unit recruitment during prolonged isometric contractions. Eur J Appl Physiol 67: 335-341, 1993.

Garland S, Griffin L, and Ivanova T. Motor unit discharge rate is no associated with muscle relaxation time in sustained submaximal contractions in humans. Neurosci Lett 239: 25-28, 1997.

Henneman E, Somjen G, and Carpenter DO. Excitability and inhibitability of motoneurons of different sizes. J Neurophysiol 28: 599-620, 1965a.

Henneman E, Somjen G, and Carpenter DO. Functional significance of cell size in spinal motoneurons. J Neurophysiol 28: 560-580, 1965 b.

Hoffer JA, Sugano N, Loeb GE, Marks WB, O'Donovan MJ, and Prat CA. Cat hindlimb motoneurons during locomotion. II. Normal activity patterns. J Neurophysiol 57: 530-552, 1987.

Johnson MA, Polgar J, Weightman D, and Appleton D. Data on the distributing of fibre types in thirty-six human muscles. An autopsy study. J Neurol Sci 18: 111-129, 1973.

Kanosue K, Yoshida M, Akazawa K, and Fuji K. The number of active motor units and their firing rates in voluntary contraction of human brachialis muscle. Jpn J Physiol 29: 427-443, 1979.
Kato M, Murakami S, Takahashi K, and Hirayama H. Motor unit activities during maintained voluntary muscle contraction at constant levels in man. Neurosci Lett 25: 149-154, 1981.

LeFever RS and De Luca CJ. A procedure for decomposing the myoelectric signal into its constituent action potentials. Pt I. Technique, theory and implementation. IEEE Trans Biomed Eng 29: 149-157, 1982.

LeFever RS, Xenakis AP, and De Luca CJ. A procedure for decomposing the myoelectric signal into its constituent action potentials. Pt II. Execution and test for accuracy. IEEE Trans Biomed Eng 29: 158-164, 1982.

Mambrito B and De Luca CJ. A technique for the detection, decomposition and analysis of the EMG signal. Electroencephalogr Clin Neurophysiol 59: 175-188, 1984.

Masakado Y, Akaboshi K, Nagata M, Kimura A, and Chino N. Motor unit firing behavior in slow and fast contractions of the first dorsal interosseous muscle of healthy men. Electroencephalogr Clin Neurophysiol 97: 290295, 1995.

Person RS. Rhythmic activity of a group of human motoneurons during voluntary contraction of a muscle. Electroencephalogr Clin Neurophysiol 36: 585-595, 1974.

Person RS and Kudina LP. Discharge frequency and discharge pattern of human motor units during voluntary contraction of muscle. Electroencephalogr Clin Neurophysiol 32: 471-483, 1972.

Rose J and McGill KC. Muscle activation and motor unit-firing characteristics in cerebral palsy. Gait Posture 13: 285-286, 2001.

Stashuk D and De Bruin H. Automatic decomposition of selective needledetected myoelectric signals. IEEE Trans Biomed Eng 35: 1-10, 1988.

Tanji $\mathbf{J}$ and Kato M. Firing rate of individual motor units in voluntary contraction of abductor digiti minimi muscle in man. Exp Neurol 40: 771-783, 1973.

Vøllestad NK, Sejersted I, and Saugen E. Mechanical behavior of skeletal muscle during intermittent voluntary isometric contractions in humans. J Appl Physiol 83: 1557-1565, 1997.

Westgaard RH and De Luca CJ. Motor unit substitution in long-duration contractions of the human trapezius muscle. J Neurophysiol 82: 501-504, 1999.

Westgaard RH and De Luca CJ. Motor control of low-threshold motor units in the human trapezius muscle. J Neurophysiol 85: 1777-1781, 2001. 